\title{
Frequency and temperature dependent attenuation constant in Barium Titanate
}

\author{
U.C. Naithani* , D.S. LingwaL, Ashok Kumar, S.C Deorani and B.S. Semwal \\ Theoretical Physics Division, H.N.B. Garhwal University Campus Pauri \\ Pauri (Garhwal), 246001 (U.P.), India.
}

\begin{abstract}
The anomalous ultrasonic attenuation $\alpha_{\mathrm{c}}$ of longitudinal waves propagating along (100) direction in Barium Titanate single crystal has been analyzed above the phase transition temperature in the frequency range $4-10 \mathrm{MHz}$ in the paraelectric phase. Critical attenuation varies as $\omega$ and $\omega^{2}$ if third - and fourth - order anharmonic interactions considered respectively. Frequency and temperature variations of attenuation are discussed.
\end{abstract}

\section{INTRODUCTION}

The ultrasonic studies have played an increasingly important role in characterizing the behaviour of a system near the co-operative phase transitions (cooperating ordering of the dipoles which gives rise to the spontaneous polarization, is destroyed by thermal agitation above the Curie temperature) and critical points. One advantage of the ultrasonic measurements is the fact that the static and the dynamic properties of the system can be simultaneously measured. Low frequency acoustic velocities provide precise information about the equilibrium adiabatic properties of the system and the effects of the temperature, pressure and external fields can be readily be

\footnotetext{
${ }^{*}$ Corresponding author
} 
studied. Ultrasonic attenuation data provide information about the dynamic behaviour and from the frequency and temperature dependences, much can be learned about the mechanisms involved.

Theoretically new ways of describing and critical phenomena in terms of fluctuation correlations have been of great importance. The large variations in the velocity and the strength of the attenuation near a transition are usually referred to 'anomalous'. Such special variations are now described as 'critical'. As revealed experimentally as well as theoretically ${ }^{1-14}$, the soft or the ferroelectric mode plays an essential role in displacive ferroelectrics. That is when the temperature approaches the paraelectric Curie-point $T_{c}$ (or in some cases structural phase transition point $T_{0}$ ), the frequency of the soft mode becomes vanishingly small resulting in an increase of its amplitude. This anomalously large amplitude should influence the acoustic mode via phonon-phonon interactions and is expected to give rise to an anomalous behavior of sound near $T_{c}$ or $T_{0}$. Barium Titanate exhibits a number of advantages over the formerly known ferroelectrics, such as: high mechanical strength to heat and moisture, presence of ferroelectric properties within a broad range of temperatures and ease of manufacturing .

Barium Titanate has attracted universal attention of researchers and has found a wide practical application. Heuter and Neuhaus ${ }^{15}$ have performed experiment and reported on sound attenuation as a function of temperature and electric field in a displacive ferroelectric $\mathrm{BaTiO}_{3}$ near $\mathrm{T}_{\mathrm{c}}$. Baluni and Naithani ${ }^{16}$ have measured the acoustical attenuation in Barium Titanate pellets. Naithani and Semwal ${ }^{17}$, Deorani et.al. ${ }^{18}$ have also derived expression for attenuation constant in displacive ferroeletrics. The aim of the present work is to study attenuation constant in $\mathrm{BaTiO}_{3}$ above the phase transition with the variation of frequency and temperature and to correlate the results with the results of other workers.

\section{CALCULATIONS}

\subsection{Temperature dependence of attenuation constant}

Ultrasonic wave is treated as a nearly static strain which modulates the equilibrium populations of the thermal phonons. Since the modes require a finite relaxation time to adjust to the new equilibrium populations, there is a phase lag with respect to the strain and an acoustic loss. In the structural phase transitions, there exists anomalously large fluctuations of the order parameter which corresponds to the excitation of certain phonon modes. These fluctuations cause anomalous absorption of the sound waves as well as shift in the velocity due to coupling of the elastic strains and the soft phonon modes. It has been shown by Tani and Tsuda ${ }^{9}$ that critical slowing down appears directly through damping. 
In the present work, we shall use the method of double-time temperature dependent Green's function which has already been applied successfully and extensively for a variety of interacting systems in the past. Most of the physical properties of the system can be expressed in terms of these Green's functions and they directly lead to thermally averaged observables. The poles of the Fourier-tranform of the Green's function give information about the elementary excitations. It is this very aspect of the Green's function method that makes them so useful.

To derive an expression for the attenuation constant, we consider the following Green's function ${ }^{19}$ for the acoustic phonons,

$$
\mathrm{G}_{\mathrm{kk}}{ }^{\prime}\left(\mathrm{t}-\mathrm{t}^{\prime}\right)=<<\mathrm{a}_{\mathrm{k}}{ }^{\mathrm{a}}(\mathrm{t}) ; \mathrm{a}_{\mathrm{k}}{ }^{\mathrm{at}}\left(\mathrm{t}^{\prime}\right)>>\text {. }
$$

Using the modified Silverman Hamilton ${ }^{20}$ (eqn.8 of reference 20) for ferroelectric perovskites. Writing the equation of motion for the Green's function (1) with the help of this modified Hamiltonian, Fourier transforming and writing it in the Dyson's equation form, one obtains ${ }^{21}$,

$$
\mathrm{G}(\omega+\mathrm{i} \varepsilon)=\delta_{\mathrm{kk}} / 2 \pi\left[\omega-\omega_{\mathrm{k}}{ }^{\mathrm{a}}+\mathrm{i} \Gamma_{\mathrm{A}}(\omega)\right]
$$

where $\Gamma_{A}(\omega)$ is the damping constant in presence of anharmonicity. Expression for $\Gamma_{\mathrm{A}}(\omega)$ in these perovskites has already been obtained in our previous studies ${ }^{18}$ and it has been shown that the presence of scattering, anharmonic and resonant interaction terms increase the value of $\Gamma_{A}(\omega)$, which is also known as the half width of the response of acoustic phonons of mode k.This increase is obtained via the stabilized soft mode frequency $\Omega$, which has an imaginary value in the harmonic approximation but is stabilized in presence of higher order anharmonic term ${ }^{22}$. If one is interested in the anomalies and temperature is not too high, the temperature dependence of $\Omega$ is given by ${ }^{17} \Omega^{2} \sim \mathrm{K}\left(\mathrm{T}-\mathrm{T}_{\mathrm{c}}\right)$, K being a temperature independent coefficient.

The attenuation constant is given by ${ }^{9}$

$$
\alpha_{\mathrm{A}}(\omega)=\Gamma_{\mathrm{A}}(\omega) / \mathrm{c},
$$

where $c$ is the sound velocity. In our previous calculations ${ }^{18}$ we have discussed $\Gamma_{A}(\omega)$ for displacive ferroelectrics and discussed the cases of $\mathrm{SrTiO}_{3}$ and $\mathrm{KTaO}_{3}$ qualitatively as well as quantitatively. The aim of the present study is to discuss the variation of attenuation constant with temperature and frequency in $\mathrm{BaTiO}_{3}$ single crystal. The temperate dependent attenuation can be expressed as

$$
\begin{aligned}
\alpha_{A}(\omega)= & {\left[A_{1}(\omega)+\left\{A_{2}(\omega)+A_{3}(\omega) /\left(T-T_{c}\right)^{1 / 2}+A_{4}(\omega) /\left(T-T_{c}\right)^{3 / 2}\right\} T+\right.} \\
& \left.\left\{A_{5}(\omega)+A_{6}(\omega) /\left(T-T_{c}\right)^{3 / 2}+A_{7}(\omega) /\left(T-T_{c}\right)^{2}\right\} T^{2}\right],
\end{aligned}
$$


where $A_{1}$ is the temperature independent term and $A_{i}{ }^{\prime s}(i=2-4)$ and $A_{j}{ }^{\prime s}(j=5-7)$ are the coefficient of $T$ and $T^{2}$ respectively $A_{i}{ }^{\prime s}$ and $A_{j}{ }^{\prime s}$ are dependent upon higher order anharmonic terms. The above expression does not give the explicit temperature dependent $\alpha_{A}(\omega)$ because of the renormalized effects. Anharmonicity is necessary in the crystal to observe these effects. It is clear from (4) that as $T \rightarrow T_{c}$, attenuation constant increases anamalously in agreement with the results of Tani and Tsuda ${ }^{9}$ and Pytte ${ }^{23}$. In the vicinity of Tc, the attenuation constant increases anamalously. However, in the low temperature range for the reduced temperature $t[=(T-T c) / T c]$, one can approximate (4) as

$$
\left.\alpha_{\mathrm{A}}(\omega) \approx \mathrm{A}_{4}(\omega)\left\{\mathrm{T} / \mathrm{T}-\mathrm{T}_{\mathrm{c}}\right)^{3 / 2}\right\}
$$

which is same as obtained by Tani \& Tsuda ${ }^{9}$. Thus in the low temperature range, the law $\alpha_{A}(\omega) \propto T /\left(T-T_{C}\right)^{3 / 2}$ can be valid to a good approximation to study these properties quantitatively.

Fig. 1 shows the raw attenuation $(\alpha)$ for longitudinal sound wave in $\mathrm{BaTiO}_{3}$ ceramic $^{24}$. In the present study only the net critical relaxation attenuation $\left(\alpha_{c}\right)$ associated with the critical fluctuations above phase transition temperature $T_{c}$ is concerned, so the remaining attenuation by extrapolating from the attenuation at much higher temperature should be subtracted as a background. The background attenuation is small, frequency independent and temperature insensitive. Taking data from reference 24 and plotting $\alpha_{c}$ $\mathrm{V}_{\mathrm{s}} \mathrm{T} /\left(\mathrm{T}-\mathrm{T}_{\mathrm{c}}\right)^{3 / 2}$ we obtain a straight line (fig.2) showing the agreement with our theoretical results in equation (5). This law is found for the reduced temperature $t=\left(T-T_{c}\right) / T_{c}$ between $1.07 \times 10^{-1}$ and $2.30 \times 10^{-1} . \alpha_{o}$ is equal to $0.4 \times 10^{2} \mathrm{dBm}^{-1}$.

Fig.1 Attenuatioin constant Vs temperature in the case of $\mathrm{BaTiO}_{3}$ single crystal (Ref. 24) 
Fig. 2 Critical attenuation Vs temperature in the case of $\mathrm{BaTiO}_{3}$ single crystal

\section{2 .2 Frequency dependence of attenuation constant}

Our previous paper ${ }^{14}$ shows that frequency dependence of attenuation is given by

$$
\alpha_{\mathrm{A}}(\omega)=\mathrm{a}_{1}+\mathrm{a}_{2} \omega^{2}+\mathrm{a}_{3} \omega,
$$

where $a_{1}$ is the constant term, independent of frequency and temperature, $a_{2}$ is a constant at a particular temperature and depends on forth-order anharmonic term, $a_{3}$ is also a constant at a particular temperature but varies with temperature and depends upon thirdorder anharmonic terms.

The $\omega^{2}$ dependence arises due to forth order anharmonic interactions, the $\omega$ dependence arises due to third order anahrmonic interaction terms. If we consider third order anharmonic interactions only, then equation (6) gives

$$
\alpha_{\mathrm{A}}(\omega) \approx \mathrm{a}_{1}+\mathrm{a}_{3} \omega
$$

Garland et.al. ${ }^{25}$ has given an expression for attenuation as,

$$
\alpha=\alpha_{0}+\alpha_{c} .
$$

Comparing equation (7) and (8), we get 


$$
\begin{aligned}
& \alpha_{0} \approx \mathrm{a}_{1} \\
& \text { and } \alpha_{\mathrm{c}} \approx \mathrm{a}_{3} \omega .
\end{aligned}
$$

Eqn (10) shows that the critical attenuation varies linearly with $\omega$, which is in prefect agreement with our previous experimental results ${ }^{16}$ and the experimental as well as theoretical results of the other workers $9,23,26,27$.

If we take fourth-order anharmonic interactions, then from eqn.(6), one gets

$$
\alpha_{\mathrm{A}}(\omega) \approx \mathrm{a}_{1}+\mathrm{a}_{2} \omega^{2} \text {, }
$$

Comparing eqn.(11) with eqn.(8), we get

$$
\begin{aligned}
& \alpha_{\mathrm{o}}=\mathrm{a}_{1} \\
& \text { and } \alpha_{\mathrm{c}} \approx \mathrm{a}_{2} \omega^{2} \text {, }
\end{aligned}
$$

where the $\omega^{2}$ dependence of attenuation is in agreement with the phemenological theory and the result of other workers ${ }^{25,28,29}$. $\mathrm{a}_{2}$ is calculated by best fit of data (ref.24) from fig (1) to equation (12). So $\mathrm{a}_{2}=4.1 \times 10^{-14} \mathrm{~S}^{2} \mathrm{~m}^{-1}$ at temperature $125^{\circ} \mathrm{C}$ and frequency $10 \mathrm{MHz}$. The variation of $\alpha_{c} V_{s} f^{2}$ is shown by us (fig.3 here).

Thus $\alpha_{\mathrm{c}}=1.62 \mathrm{f}^{2} \mathrm{dBm}^{-1}$,

where $\mathrm{f}$ is in MHz. Fig. (3) shows $\alpha_{\mathrm{c}} \mathrm{V}_{\mathrm{s}} \mathrm{f}^{2}$, which comes to be straight line in good agreement with the $\omega^{2}$ variation of the attenuation constant in the frequency range 4-10 $\mathrm{MHz}$.

Fig. 3 Criticcal Attenuatiion in BaTiO3 single crystal as a function of frequency 


\section{CONCLUSIONS}

Expression is obtained for the sound attenuation constant in Barium Titanate displacive ferroelectric single crystal with the help of Pytte's modified Hamiltonian, which includes anharmonicity upto fourth-order and using Green's function technique. In the vicinity of the phase transition temperature, the anamolously increasing sound attenuation is the consequence of the soft mode. The attenuation of sound waves is primarily due to strong interaction with thermally excited phonons in the soft mode. Attenuation constant as a function of temperature and frequency is studied. In deriving the expression for attenuation constant, different authors have used different methods, approximations and symbols. In the treatment adopted here we have used Green's function technique and the Dyson's equation treatment in presence of higher order anharmonic, resonant interaction and scattering terms in the model Hamiltonian.The Dyson's equation treatment has been found convenient to drive the shift and width of the frequency response function and hence to describe the properties of $\mathrm{ABO}_{3}$ type ferroelectric crystals. The values of constants are taken from reference 24 by best fit of data method and feeded into the expression for attenuation constant obtained by us. The results obtained are in good agreement with previous experimental and theoretical results. Anharmonicity is necessary in these crystals to observe frequency and temperature dependences of the attenuation constant.

Here we find that in the high temperature limit, attenuation varies as $T /\left(T-T_{c}\right)^{3 / 2}$. This also explains the anamolous attenuation in the vicinity of critical point $\mathrm{T}_{\mathrm{c}}$. Soft mode frequency is held responsible for the acoustical anomalies near the phase transition point.. For third order anharmonic interactions attenuation varies as $\omega$ and for forth order anharmonic interactions it varies as $\omega^{2}$. The case of a doped ferroelectric crystal will be discussed in a separete study ${ }^{30}$.

\section{ACKNOWLEDGEMENTS}

The authors are highly thankful to Dr. K.C.Purohit, Geography Department, H.N.B.Garhwal University Campus Pauri (U.P.) India, for his valuable suggestions.

\section{REFERENCES}

1. P.W.Anderson, "Proc.Int. Conference on dielectrics" ed.G.I.Skanavi, Acad. Science (Moscow, 1960).

2. W.Cocharan, "The dynamic of atoms in crystals", Edward Arnold Pub. London, 1973.

3. R.A. Cowley, Phys. Rev 134 A 981 (1964); Phys Rev. Lett. 9 159(1962); Phil.Mag. 11673.

4. W.C.Spitzer, R.C.Miller, D.A.Kleiman and L.E. Howarth, Phys.Rev.126 1710 (1962). 
5. G.Shirane, R.Nathans and V.I.Minkiewicz, Phys.Rev. 157 396(1968); Phys. Rev. Lett.19 234 (1967).

6. A.S.Barker, Jr And M.Tinkham, Phys.Rev. 1251527 (1962).

7. V.L. Gingingburg,Sov.Phys.Sol.Stat. 21829 (1960).

8. T.Kurosawa, J.Phys.Soc. Japan 161298 (1961).

9. K.Tani, Phys. Lett 25 A 400 (1967); J.Phys.Soc.Japan 2693 (1969); 29594 (1970);K.Tani and N.Tsuda 26113 (1969).

10. H.Motegi and T.Mitsui, J.Phys.Chem.Solides $\underline{25} 253$ (1964).

11. J.M.Ballantyme Phys.Rev. 136A 429 (1964).

12. J.Haroda and G.Hongo, J.Phys,Soc.Japan 2245 (1969).

13. C.Kittle, "Introduction to Solid State physics" John Wiley and sons 1966.

14. U.C.Naithani and B.S.Semwal, J.De Physique 42 C-5-659 (1981).

15. N.P.Heuter and D.P. Neuhaus, J.Acoust.Soc.Am. 27292 (1955).

16. G.N.Baluni and U.C.Naithani, Solid State Ionics(North-Holland) 22155 (1986).

17. U.C.Naithani and B.S.Semwal, Pramana (India) 11423 (1978).

18. S.C.Deorani, U.C.Naithani and B.S.Semwal, Pramana (India) $\underline{35} 181$ (1990); Int.J.Phys.Chem.Solids. (G.B.) $\underline{51} 1277$ (1990).

19. D.N.Zubarev, Sov.Phys.Usp. $\underline{3} 320$ (1960).

20. U.C.Naithani, R.P. Gairola and B.S.Semwal, J.Phy.Soc.Japan 432204 (1977).

21. B.S.Semwal and R.P.Gairola, J.Phys.Japan 42975 (1977).

22. U.C.Naithani, G.N.Balouni and B.S.Semwal, Jour.Math.Phy.Sci(India) 20 445(1986).

23. E.Pytte,Phys. Rev. B1 926 (1970).

24. See Landolt Bornstein,Series III,Vol 3 (1969),Springer Verlag,New York.

25. C.W.Garland and G.Parks,Phys.Rev.B29 221 (1984).

26.C.Mavroyami and K.N.Pathak,Phys.Rev. 182872 (1969).

27. B.Barre, K.Fossheim and K.A.Muller,Phys.Rev.Let $\underline{23} 1456$ (1969).

28. H.H.Barrett, Phys.Rev.178 743 (1969).

29. I.Toda and I.Tatsuzaki, J.Phys.Soc.Japan 311479 (1971).

30. Askok Kumar,U.C.Naithani and B.S.Semwal (in preperation). 